\title{
Cost Benefit Analysis of Four and Eight Antenatal Care Visits in Bamenda Health District, Cameroon
}

\author{
Eyvonne Ngequih Tumasang ${ }^{1,}$, , Robinson $\mathrm{Mbu}^{2}$, Wilfred Mbacham ${ }^{3}$ \\ ${ }^{1}$ Department of Health Economics Policy and Management, Faculty of Business and Management Sciences, Catholic University of \\ Cameroon, Bamenda, Cameroon \\ ${ }^{2}$ Department of Obstetrics and Gynecology, Faculty of Medicine and Biomedical Sciences, University of Yaounde 1, Yaounde, Cameroon \\ ${ }^{3}$ The Biotechnology Centre, University of Yaounde 1, Yaounde, Cameroon
}

Email address:

ntumasang@yahoo.com (E. N. Tumasang), robinsonmbu@gmail.com (R. Mbu),wfmbacham@yahoo.com (W. Mbacham)

${ }^{*}$ Corresponding author

\section{To cite this article:}

Eyvonne Ngequih Tumasang, Robinson Mbu, Wilfred Mbacham. Cost Benefit Analysis of Four and Eight Antenatal Care Visits in Bamenda Health District, Cameroon. International Journal of Health Economics and Policy. Vol. 6, No. 3, 2021, pp. $79-84$.

doi: $10.11648 /$ j.hep.20210603.11

Received: July 1, 2021; Accepted: July 20, 2021; Published: August 2, 2021

\begin{abstract}
Background: In 2016, the World Health Organization (WHO) published new antenatal care guidelines recommending an increase from four visits during pregnancy to eight contacts with skilled personnel, among other recommendations. Currently there are ongoing discussions in respect to the cost implications and potential outcomes countries can anticipate, if they make this move. Cameroon has adopted the new recommendation without analysis of the cost implication. Notwithstanding, it is imperative to investigate if pregnant women are willing to make this change, the cost implications of such changes, and if pregnant women will be willing to pay the extra cost. This study sets out to find out women's preferred number of antenatal visits, their willingness to pay for antenatal visits and to perform a cost- benefit analysis of 4 and 8 visits in Bamenda Health District (BHD). Method: An exit interview was conducted using a semi structured interviewer administered questionnaire with women attending antenatal clinic in some 14 health facilities in BHD. The health facilities were chosen purposively. A total of 396 women were interviewed. Results: Our results revealed that 177 (44.7\%) of the women preferred 4 antenatal care visits while $174(43.9 \%)$ preferred 8 antenatal care visits and $45(11.4 \%)$ preferred more than 8 antenatal visits. The extra cost for 4 and 8 visits was 10,390FCFA. The average willingness -to -pay (WTP) for 4 extra visits was calculated to be 2,209FCFA. Conclusion: Although women preferred a model of antenatal care (ANC) with 8 or more ANC contacts, they were not willing to pay for the contacts, thus it is recommended that the 4 visits model be implemented in the Bamenda Health District.
\end{abstract}

Keywords: Cost, Benefit, Four ANC Visits, Eight ANC Visits, ANC Visit, Cameroon

\section{Introduction}

Maternal health has improved in the last 25 years. Globally, maternal deaths have fallen by almost $44 \%$ since being included in the Millennium Development Goals in 1990 [1]. Although maternal mortality remains inadmissibly high, there has been an unarguable substantial drop in maternal mortality (MM) globally [2, 3]. An estimated 303,000 women died during and after pregnancy and childbirth in 2015 [1]. It is well known that almost all of these maternal deaths $(99 \%)$ occur in developing countries [1]. More than half of these deaths occurred in sub-Saharan Africa and almost one third in South Asia [1]. Generally speaking, lowincome countries still bear a large share of the global burden of maternal health problems. In 2015, Sub-Saharan Africa alone accounted for $66.3 \%$ of the world's maternal deaths [1], and there were 12 countries in this region that still had more than 500 deaths per 100,000 live births as compared to less than 5 deaths per 100,000 live births in the ten countries with the lowest maternal mortality rates [1].

In Cameroon, maternal mortality ratio (MMR) stands at $782 / 100,000$ live births [4] and it was classified as one of the countries making no progress in the achievement of the 
Millennium Development Goals [4, 5]. There has been a dropped in MM to 529/100,000 live births in 2017 still well above the figure of 1998 which was $454 / 100,000$ live births [6]. While other countries are breaking records in dropping MM figures, Cameroon is still to attain the rate it had close to 23 years ago.

Most maternal deaths are preventable, as the healthcare solutions to prevent or manage complications are well known [7]. Most of these preventable deaths during pregnancy and childbirth have been attributed to poor ANC $[8,9]$. Antenatal care is considered one of the safe motherhood interventions $[10,11]$. All women need access to ANC in pregnancy, skilled care during childbirth, and care and support in the weeks after childbirth [3]. This is in respect to the fact that ANC can serve as a platform for the delivery of highly effective health interventions that can reduce preventable maternal and newborn deaths [12].

WHO visualises a world where every pregnant woman and newborn receives quality care throughout pregnancy, childbirth and the postnatal period [2]. Within the continuum of reproductive health care, ANC provides a platform for important health-care functions, including health promotion, screening and diagnosis, and disease prevention. It has been established that by implementing timely and appropriate evidence-based practices, ANC can save lives. "Crucially, ANC also provides the opportunity to communicate with and supports women, families and communities at a critical time in the course of a woman's life" [13].

Furthermore, as indirect causes of maternal morbidity and mortality, such as human immuno-deficiency virus (HIV) and malaria infections, contribute to approximately $25 \%$ of maternal deaths [14], ANC also provides an important opportunity to prevent and manage concurrent diseases through integrated service delivery [3]. ANC helps women prepare for delivery and understand warning signs during pregnancy and childbirth. It is required to be a source of micronutrient supplementation, treatment of hypertension to prevent eclampsia, immunization against tetanus, HIV testing, in addition to medications to prevent mother-to-child transmission of HIV in cases of HIV-positive pregnant women. In areas where malaria is endemic, health personnel can also provide pregnant women with medications and insecticide-treated mosquito bed nets to help prevent this debilitating and sometimes deadly disease [15]. The objective, therefore, of ANC is to assure that every pregnancy results in the delivery of a healthy baby without impairing the mother's health [16].

However, for ANC to be more effective in preventing adverse pregnancy outcomes it should be sought early in pregnancy and should continue all through to delivery [17]. When ANC is not properly implemented, it can miss certain danger signs or delay referral to emergency obstetrical care services, therefore contributing to maternal mortality [18].

Many different approaches to ANC are used in different countries which include the traditional approach, goaldirected ANC, Focused ANC (FANC) approach and the 2016 WHO guidelines. Some countries structure and develop their approach to ANC to suit their unique circumstances; others will modify the approach from other countries while some might simply adopt an approach existing elsewhere [8]. This is the case with Cameroon that adopted the FANC approach in 2006 and has equally adopted the New WHO 2016 guideline recommending $8 \mathrm{ANC}$ contacts.

These ANC models are being changed at a point in time when problems are identified that affects the quality of the ANC services being rendered. For instance in the traditional model of ANC, pregnant women were classified as being at low risk or high risk based on predetermined criteria, this was difficult to implement effectively since women usually had at least one risk factor, even the low risk women tend to develop complications particularly during childbirth [19].

Prompted by the problem with the high risk approach (traditional model), WHO in 2002 proposed the Focused Antenatal Care (FANC) model for developing countries. WHO studies undertaken in the 1990s had suggested that a four-contact schedule was adequate [20].

FANC was aimed at addressing some of the challenges associated with the traditional model of antenatal care and to improve the quality of antenatal care services rendered [21, 22]. In this model, WHO recommends a minimum of four antenatal care visits $[21,15]$. The focus of the FANC model is centered on quality of visits rather than quantity of visits. It operates under the principle of individualization of care and views all pregnant women as being potentially at risk of developing complications thus, it aims at early identification and treatment using evidence based practice" [19].

However, in 2016 WHO instituted yet another model for ANC that recommends eight contacts instead of four visits. This recommendation was as a result of the findings that the practice and satisfaction with FANC has not been adequate and it has also been associated with lesser positive pregnancy outcome [13, 19].

An increase in the number of visits remain challenging as underutilization has been observed when the benchmark is set at four visits, eight contacts are unlikely to be feasible in low-resource settings [23].

While there are obvious benefits of increasing antenatal visits, implementing the new guidelines is going to be much more difficult to achieve given current resources constraints [24] and the financial access by the users. There is need for careful considerations of the extra challenge the new guideline poses and to understand the implications on the resources needed including among others: human resources, infrastructure, and equipment [24]. Taking into account the national disparities and inequities in access to critical health services and in health outcomes, the salient question remains whether each government should increase resources to implement the new model, or focus its efforts on strengthening the existing ANC model?

Furthermore, over half of all perinatal deaths result from deficiencies in intrapartum care, and care providers in settings with severely constrained budgets will need to consider carefully the relative benefits of investing in these additional antenatal care contacts for low risk women or 
improving the quality of intrapartum care [25].

Currently there are ongoing wrangle in respect to the cost implications and potential outcomes countries can anticipate, if they increase ANC contacts. Cameroon on its part has already adopted the new recommendation without careful analysis of the cost implication. Notwithstanding, it is imperative to investigate if pregnant women are in accord with this change; the cost implications of such changes; and if pregnant women will be willing to pay the extra cost this may entail. This paper sets out to find out women's preferred number of antenatal visits, women's willingness to pay (WTP) for antenatal visits and to do a cost benefit analysis of 4 and 8 visits in Bamenda Health District.

\section{Method}

Study design and setting

A cross sectional and analytical study was carried out in the Bamenda Health District (BHD). BHD is one of the 19 health districts in the North West Region of Cameroon. The Bamenda Health District is an urban and semi-urban area with an estimated population of 337,036 inhabitants. The district covers a total surface area of $560 \mathrm{~km}^{2}$. There is one main hospital (Bamenda Regional Hospital), which functions as a referral hospital, to many public, lay private and mission health facilities [26]. The BHD is made up of, 18 health areas, each of these health areas has one health facility that is considered as the leading health facility. The Bamenda health district is located in the Northwest Region which is the third most populated region in Cameroon, with an estimated population of more than 1.8 million inhabitants [26]. Bamenda serves as both the administrative headquarters of Mezam Division and the North West region of Cameroon. It is a cosmopolitan city made up of three towns: Mankon, Nkwen and Bamendankwe and is inhabited by people originating from all over the country, and from neighbouring countries.

Ethical clearance was obtained from the Cameroon Baptist Convention Health Board, Institutional Review Board (IRB). Administrative Clearance from the Regional Delegation of Public Health of the North West Region in Bamenda and from the heads of the different health facilities involved in the study. All participants were provided written or verbal informed consent. Pregnant women below the age of 21 were excluded from the study. Except for the inconvenience of taking time to respond to the research questionnaire, participants were not exposed to any risk. Confidentiality was ensured by coding questionnaires and protecting the database with a password. Pregnant women who fulfilled the inclusion criteria (pregnant women presenting for the second or subsequent visit), 21 years and above of age and who consented to participate in the study were enrolled into the study. Pregnant women who came for services other than ANC visits, less than 21 of age, attending ANC for the first time and those who did not give their consent were excluded.

Procedure

Between 12th February 2021 and 15th May 2021, a total of 396 pregnant women were recruited into the study based on the calculated sample size using Krejcie and Morgans table for sample size calculation [27]. Pregnant women who attended ANC in some 13 leading health facilities and at the Regional Hospital during the study period were informed on the purpose and the procedure of the study. Those who gave their consent and signed the consent form were enrolled into the study. Data was collected using a pretested interviewer administered semi-structured questionnaire with questions developed by the researcher to respond to research questions with due consultation of questionnaire design [28]. The interview was conducted when the women were exiting the health facility. Data was analysed using descriptive statistics and chi-square analysis.

\section{Results}

Characteristics of respondents

Overall, a total of 396 pregnant women gave their consent and participated in the study. The characteristics of the participants are summarized in (Table 1). A little above one third of the participants were of the 21 to 25 age group $(n=141)$ constituting $35.60 \%$ of the sample population. This was closely followed by the 26 to 30 age group $(n=138)$ making up $34.80 \%$. This result reveals that most of the pregnant women in the BHD are aged 30 and below (table 1). Almost all of the women were married $(81.8 \%)$ and had attended secondary or tertiary educational level (49.2 and $37.4 \%$ respectively). About three quarters of the women had between 0 to 2 deliveries primigravidas $(28.5 \%)$ and those who had had one delivery $(24.5 \%)$ and those with two deliveries $(21.5 \%)$. Only $106(26.8 \%)$ of the participants started ANC during the first trimester. The mean number of visits was approximately 4 although there were participants who have had up to 12 visits.

Table 1. Sociodemographic characteristics of study participants.

\begin{tabular}{lllll}
\hline Characteristics & & Frequency & Percentage & Mean \pm SD \\
\hline \multirow{4}{*}{ Age group (years) } & $21-25$ & 141 & 35.6 \\
& $26-30$ & 138 & 34.8 \\
& $31-35$ & 80 & 20.2 \\
& $36-40$ & 31 & 7.8 \\
Marital Status & ABOVE 40 & 6 & 1.5 \\
& MARRIED & 324 & 81.8 \\
& SINGLE & 68 & 17.2 \\
\hline
\end{tabular}




\begin{tabular}{lllll}
\hline Characteristics & & Frequency & Percentage & Mean \pm SD \\
\hline \multirow{3}{*}{ Level of Education } & PRIMARY & 53 & 13.4 \\
& SECONDARY & 195 & 49.2 \\
& TERTIARY & 148 & 37.4 \\
& 0 & 113 & 28.5 \\
PARITY & 1 & 97 & 24.5 \\
& 2 & 85 & 21.5 & $1.55 \pm 1.357$ \\
& 3 & 66 & 16.7 & \\
GA at first visit & 4 & 26 & 6.6 & \\
& $\geq 5$ & 9 & 2.3 & \\
\multirow{2}{*}{ Number of visits } & ABOVE 28 weeks & 25 & $6.3 \%$ & $3.82 \pm 1.721$ \\
\hline
\end{tabular}

Women's preferred number of ANC visits

Table 2. Preferred number of visits by public and private hospitals.

\begin{tabular}{|c|c|c|c|c|}
\hline VARIABLE & RESPONSE & MISSION & PUBLIC & TOTAL \\
\hline \multicolumn{5}{|c|}{ PREFERRED NUMBER OF VISITS } \\
\hline & 4 & $35(8.8 \%)$ & $142(35.9 \%)$ & $177(44.7 \%)$ \\
\hline & 8 & $53(13.4 \%)$ & $121(30.6 \%)$ & $174(43.9 \%)$ \\
\hline & MORE THAN 8 & $13(3.3 \%)$ & $32(8.1 \%)$ & $45(11.4 \%)$ \\
\hline Pearson Chi-Square & $5.579^{\mathrm{a}}$ & & $.061^{*}$ & \\
\hline
\end{tabular}

$1 \% * * * 5 \% * * 10 *$

Results on table 2 reveal that a total of $177(44.7 \%)$ respondents preferred 4 visits to 8 and more than 8 visits. Women who preferred 8 visits were slightly below (174 (43.9\%) the women who preferred 4 while $45(11.4 \%)$ of them preferred more than 8 visits. $32(8.1 \%)$ women in public hospitals compared to $13(3.3 \%)$ in private hospitals preferred more than 8 visits. Overall, the chi square results ( $\mathrm{P}$-value $=0.061)$ is not significant at $5 \%$. This implies that choice of number of visits of women did not significantly differ by the hospital types the women attended. Table 3 comparing age at first visit and the preferred choice of number of visits show that more women who went for ANC at gestational age 8 to 12 preferred 8 visits $(60)$ to 4 visits (31). Most women who went for ANC at gestational age 13 to 27 weeks (134) prefer 4 visits to 8 (104) and more than 8 visits (26). In the same light, women who went to the hospital at gestational age above 28 week prefer 4 visits (12) to 8 visits (10) and more than 8 visits (2). The chi square results $(\mathrm{P}$-value $=0.006)$ is statistically significant at $1 \%$. This implies that the choice of the number of preferred visits significantly depended on the gestational age at first ANC. Women who went for ANC at a later gestational age preferred 4 visits while more of those who went for ANC earlier preferred 8 and above 8 visits.

Table 3. Gestational age at first visit against preferred number of visits.

\begin{tabular}{llllll}
\hline & & \multicolumn{2}{l}{ Preferred number of visits } & \\
\cline { 3 - 4 } & & $\mathbf{4}$ visits & 8visits & More than 8 & 25 (6.3\%) \\
GA AT FIRST VISIT & $\geq 28$ weeks & $12(3.0 \%)$ & $10(2.5 \%)$ & $3(0.8 \%)$ & \\
& 8 TO 12weeks & $31(7.8 \%)$ & $60(15.2 \%)$ & $15(3.8 \%)$ & $106(26.8 \%)$ \\
Pearson Chi-Square & 13 TO 27weeks & $134(33.4 \%)$ & $104(26.3 \%)$ & $26(6.6 \%)$ & $264(66.8 \%)$ \\
\hline
\end{tabular}

The Cost-Benefit of 4 and 8 ANC visits for women in the BHD

Table 3: Cost and Benefit of 4 and 8 visits

Table 4. COST BENEFIT ANALYSIS

\begin{tabular}{llll}
\hline Variables & FIRST VISIT & SUBSEQUENT VISITS & Total \\
\hline FIRST 4 VISITS & 17799.24 & $2597.60 * 3$ & 25592 \\
EXTRA 4 VISITS & & $2597.60 * 4$ & 10390 \\
8 VISIT & & & 35,982 FCFA \\
WILLINGNESS TO PAY FOR EXTRA 4 VISIT & & 2209.07 \\
\hline
\end{tabular}


The average total cost for 4 visits was calculated to be 25,592 FCFA while that of 8 visits was 35,982 FCFA. This gives an extra cost for 4 visits to be 10,390 FCFA. The average willingness to pay for 4 extra visits was 2,209 FCFA. Considering the fact that the extra cost for four additional ANC visit is far higher than what the women are willing to pay for these extra visits, and that this cost will increase when other factors such as transportation and waiting times at the health facility are taken into consideration, we concluded that 8 visits is not cost beneficial for pregnant women in the BHD.

\section{Discussion}

This paper intended to find out women's preferred number of antenatal visits, women's willingness to pay (WTP) for antenatal visits and to do a cost benefit analysis of 4 and 8 visits in Bamenda Health District. Only about one quarter $(26.8 \%)$ of the women started ANC within the first trimester of their pregnancy this finding is similar to that of other authors [26, 29, 30, 31] who found out that most women usually begin ANC late because they do not know its benefit; limited access to facility; uncertainty about pregnancy and reluctance to go for many visits.

There was no significant difference in the preferred number of visits, as $44.7 \%$ of the women who constituted about half of the sample population preferred 4 visits. The women who preferred 8 and more visits when combined made up $55.3 \%$ of the study population this is contrary to the findings [13] that women desire more ANC visits in order to experience a positive pregnancy outcome.

In this study we realized that the majority of the women who preferred 4 visits started ANC late. This is probably to reduce the number of ANC visits they will have before delivery. Conversely, most of the women who initiated ANC during the first trimester preferred 8 or more visits. Will this disparity be addressed if the number of visits is increased from 4 to 8 ? This question stands valid moreso in this study where $130(32.9 \%)$ of the women who preferred 8 or more visits initiated $\mathrm{ANC}$ late, with $6.9 \%$ of them beginning ANC in the third trimester.

As would be expected, the cost of 8 visits was 10390 FCFA more than the cost for 4 visits. This is where the dispute is if women will be able to afford this extra cost. In this present study women's willingness to pay for the extra 4 visits was only 2209 FCFA. This led to the conclusion that the implementation of the 8 visits is not cost beneficial to these women. Although the researchers did not come across any studies that looked at cost benefit of ANC, in a study carried out in the Noun division of the West Region of Cameroon [32], pregnant women without sufficient financial resources had to spend long hours waiting to be attended to; while some die due to negligence of the professionals. Meanwhile, the same study revealed that clients who are financially viable are able to pay extra to receive prompt, careful, and effective care [32]. This buttresses the idea that without money no matter the number of times that the women show up for ANC their experience of it will be poor. Financial constraints have been a major set back to ANC attendance [33], more especially in Cameroon where payment for health services is largely out-of-pocket [34].

\section{Conclusion}

Although there are benefits associated with the 8 contacts recommended by $\mathrm{WHO}$ and our findings revealed women preferred a model of ANC with 8 or more ANC contacts, the 8 contacts are not cost beneficial and women are not willing to pay for the contacts. Thus, we recommend that the 4 visits model be implemented in the Bamenda Health District.

\section{Recommendation for Further Research}

This study used only the user fee to do a cost benefit analysis with. A study with a more detailed estimate of cost is recommended to obtain a clear picture of cost of ANC in the Bamenda Health District.

\section{References}

[1] Alkema L, Chou D, Hogan D, Zhang S, Moller A-b, Gemmill A, Fat DM, Boerma T. (2015). Global, regional, and national levels and trends in maternal mortality between 1990 and 2015, with scenario-based projections to 2030: a systematic analysis by the UN maternal mortality estimation inter-agency Group. Lancet.; 6736: 1-13.

[2] Tuncalp Ö, Were WM, MacLennan C, Oladapo OT, Gulmezoglu AM, Bahl R, Daelmans B, Mathai M, Say L, Kristensen F, $€$ Temmerman M, Bustreo F. (2015). Quality of care for pregnant women and newborns - the WHO vision. BJOG; 122: 1045-1049.

[3] WHO. (2018). Maternal Mortality.

[4] Verla, ET, Ojong-Alasia, M., Sama, J., Tumasang, E Ndipowa, J., Atanga, M. (2016). "Various Health Care Providers' Knowledge of the Partogram Use during Childbirth, at the Bamenda Health District, Cameroon", Asian Journal of Medicine and Health.

[5] Institut National de la Statistique de la République du Cameroun. (2011). Enquête Démographique et de Santé et à Indicateurs Multiples. Yaounde (Cameroon). Retrieved from http://dhsprogram.com/pubs/pdf/FR260/FR260.pdf.

[6] Meh, C; Thind, A. and Terry, AL. (2020). Ratios and determinants of maternal mortality: a comparison of geographic differences in the northern and southern regions of Cameroon. Meh et al. BMC Pregnancy and Childbirth 20: 194 https://doi.org/10.1186/s12884020-02879-y accessed June 15, 2020.

[7] Finlayson, K. W. and Downe, S. 2013. Why do women not use antenatal services in low and middle income countries? A metasynthesis of qualitative studies. PLoS Medicine, 10 (1): e1001373. doi: 10.1371/journal.pmed.1001373. 
[8] Ngxongo, TSP. (2015). Implementation of the Basic Antenatal Care approach: A tailored practice framework for eThekwini District, KwaZulu-Natal. PhD thesis.

[9] Ngxongo, TSP. (2019). "Chapter 1 Basic Antenatal Care Approach to Antenatal Care Service Provision", Intech Open.

[10] Prata N, Sreenivas A, Greig F, Walsh J, Potts M. (2010). Setting priorities for safe motherhood interventions in resource-scarce settings. Health Policy; 94: 1-13.

[11] Hitimana R. Lindholm L., Krantz G., Nzayirambaho M. and Pulkki-Brännström, A. (2018). Cost of Antenatal care for the health sector and for households in Rwanda. BMC Health Services Research.

[12] Saad-Haddad, G., DeJong, J., Terreri, N., Restrepo-Méndez, M., Perin, J., Vaz, L., Newby, H., Amouzou, A., Barros, A. and Bryce, J. (2016). Patterns and determinants of antenatal care utilization: analysis of national survey data in seven countdown countries. J Glob Health. 6 (1): 010404.

[13] World Health Organization. (2016). WHO Recommendations on Antenatal Care for a positive pregnancy experience. Geneva, Switzerland.

[14] Souza JP, Gülmezoglu AM, Vogel J, Carroli G, Lumbiganon P, Qureshi Z et al (2013). Moving beyond essential interventions for reduction of maternal mortality (the WHO Multi-country Survey on Maternal and Newborn Health): a cross sectional study. Lancet. 381 (9879): 1747-55. doi: 10.1016/S0140-6736(13)60686-8.

[15] UNICEF Cameroon Country Programme 2018-2020 Strategy Note Cameroon Health Programme 2018-2020. Available at: http://files.unicef.org/transparency/documents/Cameroon Stra tegy $\% 20$ Note $\% 20-\% 20$ Health\%20-\%20final\%20sd.pdf.

[16] Ekabua, J., Ekabua, K. and Njoku, C. 2011. Proposed framework for making focussed antenatal care services accessible: a review of the Nigerian setting. ISRN Obstetrics and Gynecology. doi: 10.5402/2011/253964 ID 253964.

[17] Chorongo, D., Okinda, F., Kariuki, E., Mulewa, E., Ibinda, F., Muhula, S., Kimathi, G., andMuga, R. (2016). Factors influencing the utilization of focused antenatal care services in Malindi and Magarini sub-counties of Kilificounty, Kenya. The Pan African Medical Journal. 25 (Supp 2): 14. doi: 10.11604/pamj.supp.2016.25.2.10520.

[18] Manzi, A., Nyirazinyoye, L, Ntaganira, J., Magge, H., Bigirimana, E., Mukanzabikeshimana, L, Hirschhorn LR., and Hedt-Gauthier, B. (2018). Beyond coverage: improving the quality of antenatal care delivery through integrated mentorship and quality improvement at health centers in rural Rwanda. BMC Health Services Research. 18: 136 https://doi.org/10.1186/s12913-018-2939-7.

[19] Oshinyemi1, T., Aluko, J. and Oluwatosin, O. (2018). Focused antenatal care: Re-appraisal of current practices. International Journal of Nursing and Midwifery. Vol. 10 (8), pp. 90-98. DOI: 10.5897/IJNM2018.0312 [accessed on 22 Jan 2019].

[20] WHO. (2001). WHO antenatal care randomized trial: manual for the implementation of the new model. Geneva: World Health Organization, 2001. www.who.int/entity/reproductivehealth/publications/maternal _perinatal_health/RHR_01_30/en/.
[21] World Health Organization (2002). Antenatal Care Randomized Trial: Manual for the Implementation of the New Model. Geneva. http://apps.who.int/iris/handle/10665/42513.

[22] Olamijulo JA, Oluwole AA, Babah OA, Aderolu MB, Okunola HA, Sanusi MM (2015). Acceptability of Focused Antenatal care by pregnant Nigerian Women and Factors Influencing it. Tropical Journal of Obstetrics and Gynaecology 32 (2): 90-96 [accessed on 22 Jan 2019].

[23] Mchenga, M. Burger, R. and von Fintel, D. (2019). Examining the impact of WHO's Focused Antenatal Care policy on early access, underutilisation and quality of antenatal care services in Malawi: a retrospective study. Mchenga et al. BMC Health Services Research (2019) 19: 295 https://doi.org/10.1186/s12913-019-4130-1.

[24] AFIDEP. (2016) Implications of new WHO antenatal guidelines on maternal mortality.

[25] Weeks A, Temmerman M. (2016). New WHO antenatal care model—quality worth paying for? Lancet. 388: 2574-5.

[26] Warri D. and George, A. (2020). Perceptions of pregnant women of reasons for late initiation of antenatal care: aqualitative interview study. BMC Pregnancy and Childbirth. 20: 70 https://doi.org/10.1186/s12884-020-2746-0.

[27] Krejcie, R. V., \& Morgan, D. W., (1970). Determining Sample Size for Research Activities. Educational and Psychological Measurement.

[28] Siniscalco MT, Auriat N. (2005). Questionnaire design. In: Ross K, Quantitative research methods in educational planning. Paris. UNESCO. Google Scholar.

[29] Myer L, Harrison A. (2003). Why do women seek antenatal care late? Perspectives from rural South Africa. J Midwifery Women's Health. 48 (4): 268-272. PubMed | Google Scholar.

[30] Halle Ekane GE et al. (2015b) Perceptions of antenatal care services by pregnant women attending government health centres in the Buea Health District, Cameroon: a cross sectional study. Pan African Medical Journal. 2015; 21: 45. [doi: 10.11604/pamj.2015.21.45.4858].

[31] Ebonwu J, Mumbauer A, Uys M, Wainberg ML, MedinaMarino A (2018). Determinants of late antenatal care presentation in rural and peri-urban communities in South Africa: A cross-sectional study. PLoS ONE 13 (3): e0191903. https://doi.org/10.1371/journal.pone.0191903.

[32] Moungbakou, IBM. (2018). Commodification of care and its effects on maternal health in the Noun division (West Region - Cameroon) BMC Medical Ethics, 19 (Suppl 1): 43 https://doi.org/10.1186/s12910-018-0286-1.

[33] Tolefac, PN; Halle-Ekane, GE; Agbor, VN; Sama, CB; Ngwasiri, C and Tebeu, PM (2017). Why do pregnant women present late for their first antenatal care consultation in Cameroon? Maternal Health, Neonatology, and Perinatology DOI 10.1186/s40748-017-0067-8.

[34] UNICEF. (nd). Cameroon Country Programme 2018-2020 Strategy Note Cameroon Health Programme 2018-2020. http://files.unicef.org/transparency/documents/Cameroon_Stra tegy $\% 20$ Note $\% 20-\% 20$ Health\%20-\%20final\%20sd.pdf. 